\title{
Protease Inhibitor Formulation LB1148
}

National Cancer Institute

\section{Source}

National Cancer Institute. Protease Inhibitor Formulation LB1148. NCI Thesaurus. Code C137683.

A proprietary formulation comprised of the serine protease inhibitor tranexamic acid (TXA), polyethylene glycol (PEG), electrolytes, and sugar, with potential Gl protective activity. After reconstitution in water and upon oral or enteral administration before abdominal surgery, TXA in the protease inhibitor formulation LB1148 targets and inhibits several serine proteases in the gastrointestinal (Gl) tract, thereby preventing and protecting the intestinal mucosa against degradation, which preserves gastrointestinal (Gl) integrity, function and motility. By inhibiting digestive proteases, the intestinal mucosal barrier is preserved during acute physiologic shock, which may prevent multiorg an failure, postoperative ileus and surgical intra-abdominal adhesions. PEG enhances transport of TXA through the Gl tract. The other components provide energy and balance dietary electrolytes, thereby promoting healing of the GI barrier. 